\title{
Sphenoparietal sinus transposition technique: optimization of the surgical corridor with preservation of prominent bridging veins between the brain and the cranial base during aneurysm clipping via the pterional approach
}

\author{
Takeya Niibo, MD, ${ }^{1}$ Katsumi Takizawa, MD, ${ }^{1}$ Jurou Sakurai, MD, ${ }^{1}$ Seizi Takebayashi, MD, PhD, ${ }^{1}$ \\ Hiroyasu Koizumi, MD, PhD, ${ }^{1}$ Toru Kobayashi, MD, PhD, ${ }^{1}$ Rina Kobayashi, MD, ${ }^{1}$ \\ Kouta Kuris, MD, PhD, ${ }^{1}$ Syusuke Gotou, MD, ${ }^{1}$ Ryousuke Tsuchiya, MD, ${ }^{1}$ and \\ Hiroyasu Kamiyama, MD²
}

${ }^{1}$ Department of Neurosurgery, Asahikawa Red Cross Hospital, Asahikawa; and 2Department of Neurosurgery, Sapporo Teishinkai Hospital, Sapporo, Japan

\begin{abstract}
OBJECTIVE The sylvian bridging veins between the brain and the dura on the inner surface of the sphenoid wing can restrict brain retraction for widening of the lateral retrocarotid space during clipping surgery for internal carotid artery (ICA)-posterior communicating artery ( $\mathrm{PCOA}$ ) and basilar apex (BX) aneurysms. In such cases, the authors perform extradural anterior clinoidectomy with peeling of the temporal dura propria from the periosteal dura and inner cavernous membrane around the superior orbital fissure, with the incision of the dura mater stretching from the base of the temporal side to just before the distal dural ring of the ICA (termed by the authors as the sphenoparietal sinus transposition [SPST] technique). This technique displaces the bridging segment of the sylvian vein posteriorly and enables widening of the surgical space without venous injury. In this study, the authors observed the operative nuances and investigated the usefulness of this technique.
\end{abstract}

METHODS The authors retrospectively reviewed the medical charts of 66 consecutive patients with ICA-PCoA and BX aneurysms between January 2016 and July 2018. This technique was performed in 8 patients (5 patients with PCoA aneurysms and 3 with $B X$ aneurysms) in whom the bridging segments of the sylvian veins between the brain and the skull base restricted brain retraction for widening of the surgical space. The surface areas of the lateral retrocarotid space and the aneurysm were measured at the most visible working angle before and after the SPST technique was performed.

RESULTS With the use of the SPST technique, an adequate surgical space for aneurysm clipping was obtained with preservation of the bridging veins in all patients. The mean surface areas of the lateral retrocarotid space $(p=0.002)$ and aneurysm ( $p=0.001$ ) were significantly increased from $18.3 \pm 18.8$ and $2.8 \pm 2.5 \mathrm{~cm}^{2}$ before to $64.2 \pm 21.1$ and $20.9 \pm$ $20.6 \mathrm{~cm}^{2}$, respectively, after the SPST technique was performed.

CONCLUSIONS The SPST technique enables displacement of the bridging segments of the sylvian veins without venous injury and enables widening of the surgical space around the lateral retrocarotid area.

https://thejns.org/doi/abs/10.3171/2020.1.JNS192823

KEYWORDS anterior clinoidectomy; anterior clinoid process; paraclinoid; skull base; vascular disorders

$\mathrm{T}$ 7 HE pterional-transsylvian approach is one of the most versatile and commonly performed approaches during clipping surgery for intracranial aneurysms. Internal carotid artery (ICA)-posterior communicating artery $(\mathrm{PCoA})$ aneurysms are typically repaired via the pterional-transsylvian approach. ${ }^{1}$ In addition, this approach is frequently used in clipping surgery for upper basilar artery aneurysms. ${ }^{2,3}$ In clipping surgery for these aneurysms, the surgical corridor involves the lateral retrocarotid space, which is accessed via the space between the

ABBREVIATIONS ACP = anterior clinoid process; $B X=$ basilar apex; DMCV = deep middle cerebral vein; FBBV = frontobasal bridging vein; fSSV = superficial sylvian vein with frontal lobe tributaries; ICA = internal carotid artery; $M O B=$ meningo-orbital band; PCOA = posterior communicating artery; SOF = superior orbital fissure; SPS = sphenoparietal sinus; SPST = sphenoparietal sinus transposition; SSV = superficial sylvian vein; TPV = temporopolar vein; tSSV = superficial sylvian vein with temporal lobe tributaries. SUBMITTED October 16, 2019. ACCEPTED January 13, 2020.

INCLUDE WHEN CITING Published online March 20, 2020; DOI: 10.3171/2020.1.JNS192823. 
skull base and brain. The superficial sylvian veins (SSVs), which represent one of the three dominant venous drainage systems of the lateral cerebrum, usually course from the brain to the dural sinus along the sphenoid wing to drain into the sphenoparietal sinus (SPS) ${ }^{4}$ In other words, these veins can pose an obstacle for accessing aneurysms. Therefore, the bridging veins are occasionally sacrificed during dissection. ${ }^{5,6}$ Although clinical complications caused by intracranial venous sacrifice are rare, ${ }^{7}$ sacrifice of these veins can cause venous congestion, hemorrhage, and edema in cases in which the sacrificed veins that are damaged during surgery are involved in major drainage pathways. ${ }^{6,89}$ At present, there is no established method for predicting the consequences of sacrificing the veins. Thus, surgeons should aim to preserve intracranial veins while reserving a wide enough space within the surgical field for safe manipulation.

We retrospectively reviewed 8 cases of ICA-PCoA and basilar apex (BX) aneurysms treated with clipping surgeries via the pterional-transsylvian approach, in which the bridging segment of the sylvian veins between the brain and the skull base restricted the ability to obtain adequate surgical spaces for aneurysm clipping, while sacrifice of these veins was considered to be associated with high risks for venous complications. In all of these patients, we used a technique that enabled displacement of the bridging segment of the sylvian veins posteriorly without venous injury, which resulted in obtaining adequate surgical corridors. We have termed this maneuver the "sphenoparietal sinus transposition (SPST) technique." In this report we describe the operative nuances of this technique and our investigation of the validity of this method for widening of the surgical corridor by quantifying the exposure enhancement of the surgical working area.

\section{Methods}

The present study was preapproved by the institutional review board at our institution.

\section{Patient Population}

We retrospectively reviewed all cases of ICA-PCoA and BX ruptured and unruptured aneurysms surgically treated at our institution from January 2016 to July 2018. This review resulted in 66 patients (59 patients with ICAPCoA and 7 patients with BX aneurysms) treated with surgical clipping via the pterional-transsylvian approach. In 8 of these 66 patients (12\%), approaching the lateral retrocarotid space was limited by the bridging sylvian veins between the brain and the dura on the inner surface of the sphenoid bone. The SPST technique was utilized for these 8 patients in order to obtain adequate surgical corridors without sacrificing these veins.

Possible adverse events associated with the SPST technique were evaluated according to postoperative complications and results of CT and MRI studies.

\section{Operative Technique}

We employed the distal transsylvian approach to preserve the sylvian veins and their tributaries. ${ }^{10}$ If a promi- nent bridging vein between the brain and the dura on the inner surface of the sphenoid bone obstructed brain retraction for widening of the surgical space (Figs. 1-4), the arachnoid membrane that wrapped the vein was peeled away from the vein to make the veins more stretchable (denuding technique). ${ }^{5}$ If the brain retraction was still restricted even after these manipulations, we utilized the SPST technique.

The SPST technique is described here and shown in Video 1.

VIDEO 1. SPST technique. The sylvian vein segments bridging between the brain and the skull base restricted brain retraction for widening of the retrocarotid space. In addition, brain retraction caused bleeding from the dural entrance of the sylvian vein. Therefore, we returned to the epidural space and unroofed the lesser wing of the sphenoid bone overlying the superior orbital fissure (SOF) and the inferolateral wall of the SOF around the meningo-orbital band (MOB). The MOB was cut and the dura propria was peeled from the periosteal dura and inner cavernous membrane until the anterior clinoid process (ACP) was exposed. The ACP was removed with microrongeurs in a piecemeal fashion. The incision of the dural flap was stretched from the temporal base side to the distal dural ring of the ACP. The bridging segment of the sylvian veins combined with the dural entrance of these veins is displaced posteriorly and the retrocarotid space is markedly widened. Copyright Asahikawa Red Cross Hospital. Used with permission. Click here to view.

The following procedures were carried out under microscopic magnification. The frontal dura was elevated from the anterior cranial fossa, and the orbital roof was flattened with a power drill to gain a wider working space. The temporal dura was dissected from the temporal bone until the lateral margin of the superior orbital fissure (SOF) was exposed. The roof of the SOF was skeletonized and the meningo-orbital band (MOB) was exposed (Fig. 1B). The lesser wing of the sphenoid bone overlying the SOF and the inferolateral wall of the SOF, which is part of the greater wing of the sphenoid bone, was unroofed with a power drill. This step provides mobility for the MOB and enables dissection of the dura propria from the lateral inferior aspect of the anterior clinoid process (ACP) with a microdissector (Fig. 1C). After these maneuvers, it was possible to identify a cleavage plane that developed between the frontal dura propria and the temporal dura propria at the MOB (Fig. 1D). This structure allows estimation of the thickness of the dura propria and the correct layer between the dura propria and the periosteal dura. Peeling of the temporal dura propria from the periosteal dura was then started at this cleavage plane of the MOB and continued until the medial aspect of the ACP was exposed (Fig. 1E). Care was taken to maintain the sphenoparietal sinus (SPS) on the side of the dura propria during peeling of the dura propria (Fig. 2C). The incision of the dural flap was stretched from the base of the temporal side to the tip of the ACP, with the dural entrance of the bridging veins maintained outside of the dural flap (Fig. 1F) to facilitate the exposure of the tip of the ACP. After dissection of the dura propria from the ACP (Fig. $1 G)$, the ACP was removed with bone rongeurs of various sizes in a piecemeal fashion (Fig. 1H). No power drill was used for removal of the ACP in our series. After removal of the tip of the ACP (Fig. 1I), the incision of the dural 

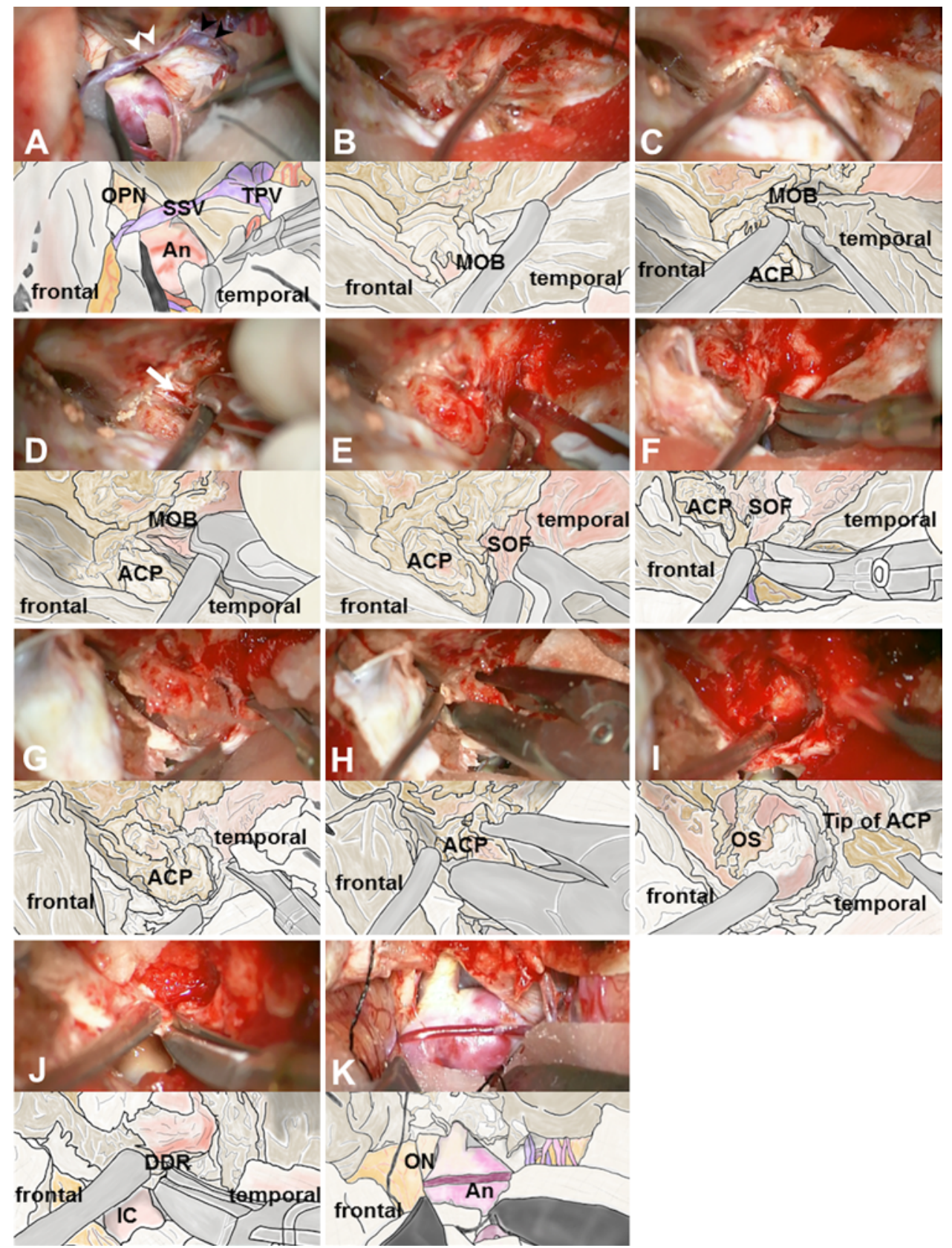

FIG. 1. Operative surgical steps for the SPST technique. Microscopic views combined with schematic illustrations during clipping of the right ICA-PCOA aneurysm. A: A microscopic view before the SPST technique was performed. The white arrowheads indicate the SSV, and the black arrowheads indicate the TPV. The short bridging segment of these veins restricted brain retraction for widening of the surgical space. B: Exposure of the MOB. C: Dissection of the dura propria from the lateral inferior aspect of the ACP. D: Initiation of the peeling of the temporal dura propria from a cleavage plane between the frontal and temporal dura propria at the MOB (solid white arrow). E: Peeling of the dura propria continues in the direction of the medial temporal side. F: Stretching of the incision of the temporal dural flap from the base of the temporal base in the direction of the tip of the ACP for exposure of the tip of the ACP. G: The ACP is dissected from the dura propria and exposed subtotally. H: Removal of the ACP with a microrongeur in a piecemeal fashion. I: Removal of the tip of the ACP. J: Stretching of the incision of the temporal dural flap until the distal dural ring of the ICA is exposed. $\mathrm{K}$ : Completion of the SPST technique. The bridging part of the SSV and TPV combined with the dural entrance of these veins is displaced posteriorly and the retrocarotid space is markedly widened. $A n=$ aneurysm; DDR = distal dural ring; IC = internal carotid artery; ON, OPN = optic nerve; OS = optic strut; SOF = superior orbital fissure. Schematic illustrations copyright Takeya Niibo. Used with permission. 

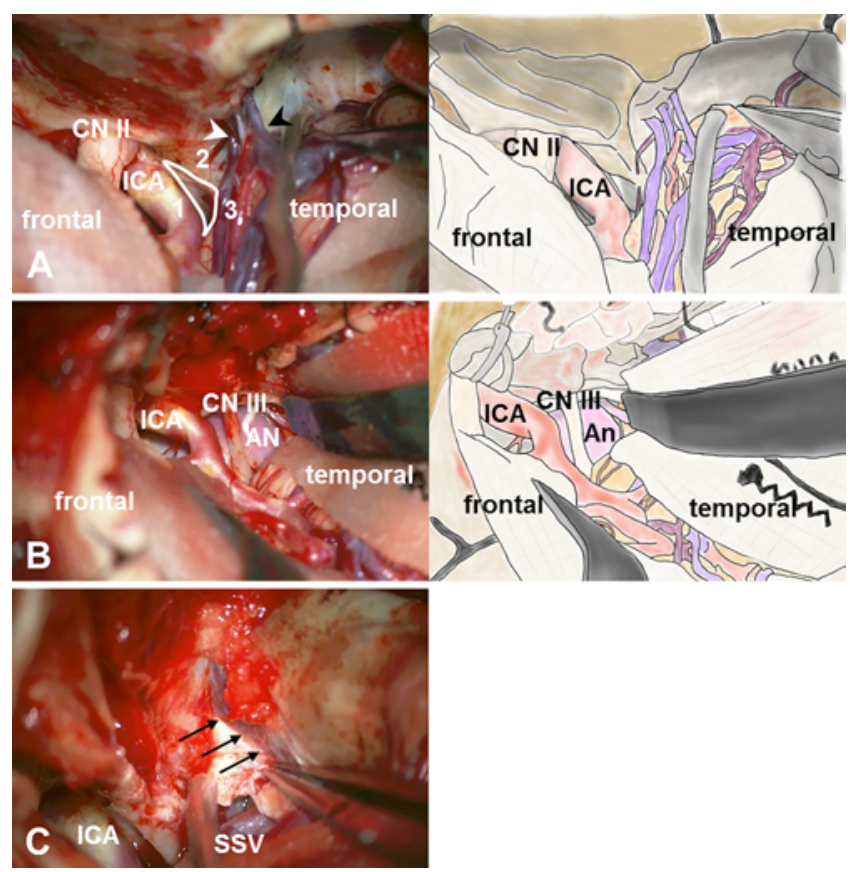

FIG. 2. Intraoperative views of clipping surgery for a BX aneurysm. A: Microscopic view (left) combined with a schematic illustration (right) before the SPST technique was performed. The white arrowhead indicates the SSV, and the black arrowhead indicates the TPV. Surface landmarks show the lateral retrocarotid space (white outline; 1 , the lateral aspect of the ICA; 2 , the medial aspect of the tentorial edge; and 3 , the margin of the frontal and temporal lobes). These veins with short bridging segments restrict temporal lobe retraction for widening of the surgical space. B: Microscopic view (left) combined with a schematic illustration (right) after the SPST technique was performed. The bridging segments of the SSV and the TPV combined with the dural entrance of these veins have been displaced posteriorly, and the temporal lobe is retracted safely. Schematic illustrations in panels A and B copyright Takeya Niibo. Used with permission. C: Microscopic view during peeling of the temporal dura propria. The solid black arrows indicate the visible course of the sphenoparietal sinus at the surface of the temporal dura. $\mathrm{AN}, \mathrm{An}=$ aneurysm; $\mathrm{CN} \| \mathrm{I}=$ optic nerve; $\mathrm{CN} \mathrm{II}=$ o oculomotor nerve.

flap was stretched until just before the distal dural ring of the ICA (Fig. 1J). With the use of these techniques, the bridging veins in combination with the dural entrance of these veins were effectively displaced posteriorly without venous injury (Fig. 1K).

\section{Measurements}

The working area was defined as the area that was visualized at surgery, and we were able to perform surgical maneuvers in this area. To evaluate the widening of the surgical corridor afforded by the SPST technique, we measured the three following parameters using intraoperative videos with safe brain retraction at the most visible working angle just before and after the SPST technique was performed: 1) the surface area of the lateral retrocarotid space, 2) the surface area of the aneurysm, and 3) the length of the ICA on its lateral aspect. We defined the working area as the lateral retrocarotid space because it is a vital space for aneurysm clipping. The lateral retrocarotid space was defined as the surrounding area, with three
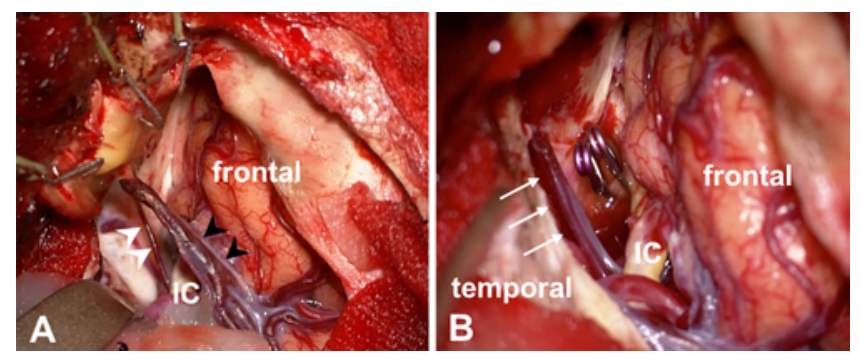

FIG. 3. Intraoperative views of clipping surgery for a left ICA-PCOA aneurysm. A: Microscopic view before the SPST technique was performed. The black arrowheads indicate the SSV, and the white arrowheads indicate the DMCV. These veins course across the operative field and restrict attainment of an adequate surgical space. B: Microscopic view after the SPST technique was performed. The bridging veins are displaced posteriorly (white arrows) and the surgical space is widened.

anatomical landmarks as follows: the lateral aspect of the ICA, the medial aspect of the tentorial edge, and the margin of the frontal and temporal lobes (Fig. 3A). To calculate these parameters, the segmented lines were delineated in the region of interest by two experienced neurosurgeons (T.N. and J.S.), and measurements were performed with the aid of open source software (ImageJ, version 1.52e, National Institutes of Health).

\section{Statistical Analyses}

Data were processed using commercially available software (SPSS version 21.0, IBM Corp.). The paired Mann-Whitney U-test was used to compare surface areas of the retrocarotid space and aneurysms as well as the length of the lateral aspect of the ICA before and after the SPST technique was performed.

\section{Results}

Of 8 patients ( 5 men and 3 women, aged 53-84 years, median age 70 years; Table 1 ) who underwent surgery performed with the SPST technique, 3 patients had an unruptured ICA-PCoA aneurysm, 2 patients had a ruptured ICA-PCoA aneurysm, and 3 patients had an unruptured BX aneurysm.
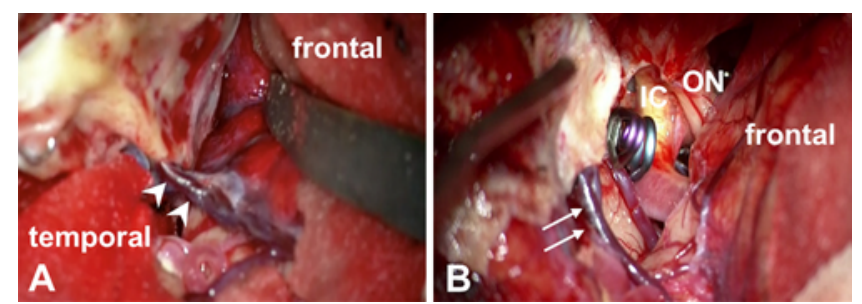

FIG. 4. Intraoperative views of clipping surgery for a left ICA-PCoA aneurysm. A: Microscopic view before the SPST technique was performed. The white arrowheads indicate the SSV, which obstructs widening of the space between the brain and the skull base. B: Microscopic view after the SPST technique was performed. The bridging segment of the SSV in combination with the dural entrance is displaced posteriorly (white arrows). 
TABLE 1. Characteristics of 8 patients who underwent the SPST technique for aneurysm clipping

\begin{tabular}{|c|c|c|c|c|c|c|c|c|c|c|}
\hline \multirow{2}{*}{$\begin{array}{l}\text { Case } \\
\text { No. }\end{array}$} & \multirow[b]{2}{*}{ Sex } & \multirow{2}{*}{$\begin{array}{l}\text { Age, } \\
\text { Yrs }\end{array}$} & \multirow{2}{*}{$\begin{array}{l}\text { Aneurysm } \\
\text { Site }\end{array}$} & \multirow{2}{*}{$\begin{array}{c}\text { Veins Blocking } \\
\text { Op Corridor }\end{array}$} & \multicolumn{2}{|c|}{ Retrocarotid Space Area, $\mathrm{cm}^{2}$} & \multicolumn{2}{|c|}{ Exposed Aneurysm Area, $\mathrm{cm}^{2}$} & \multicolumn{2}{|c|}{ ICA Lateral Aspect Length, mm } \\
\hline & & & & & Pre-SPST & Post-SPST & Pre-SPST & Post-SPST & Pre-SPST & Post-SPST \\
\hline 1 & M & 74 & $\mathrm{PCOA}$ & fSSV, DMCV & 33.02 & 74.43 & 3.78 & 10.34 & 8.73 & 14.71 \\
\hline 2 & M & 75 & PCoA & fSSV, TPV & 10.89 & 88.61 & 7.62 & 66.02 & 4.68 & 13.84 \\
\hline 3 & $\mathrm{~F}$ & 69 & PCoA & fSSV, FBBV & 9.28 & 47.73 & 0.53 & 7.26 & 3.73 & 11.91 \\
\hline 4 & $\mathrm{~F}$ & 84 & PCoA & fSSV, tSSV & 12.09 & 56.30 & 4.44 & 12.58 & 8.23 & 14.05 \\
\hline 5 & $\mathrm{~F}$ & 70 & PCoA & fSSV & 5.37 & 23.20 & 2.91 & 14.48 & 2.78 & 8.90 \\
\hline 6 & M & 69 & $\mathrm{BX}$ & tSSV & 5.36 & 68.54 & 0 & 6.1 & 7.11 & 16.37 \\
\hline 7 & $M$ & 69 & $B X$ & tSSV, TPV & 14.72 & 72.85 & 1.12 & 36.3 & 15.04 & 21.99 \\
\hline 8 & M & 53 & $B X$ & FBBV & 59.94 & 82.07 & 2.25 & 13.89 & 12.90 & 17.42 \\
\hline
\end{tabular}

\section{Pattern of Venous Interference and Effect of the SPST Technique}

In the 8 patients in whom the SPST technique was performed, the bridging segment of the sylvian veins was relatively short, and brain retraction for accessing the lateral retrocarotid space could have easily caused avulsion and/or tension injuries in these veins at the dural entrance on the sphenoid wing (Figs. 1-4). The bridging veins that restricted brain retraction were as follows: a superficial sylvian vein (SSV) with frontal lobe tributaries (fSSV) in 1 patient with an ICA-PCoA aneurysm; an SSV with temporal lobe tributaries (tSSV) in 1 patient with a BX aneurysm; a frontobasal bridging vein (FBBV) in 1 patient with a BX aneurysm; both an fSSV and a deep middle cerebral vein (DMCV) in 1 patient with a PCoA aneurysm; both an fSSV and a temporopolar vein (TPV) in 1 patient with a PCoA aneurysm; both an fSSV and an FBBV in 1 patient with a PCOA aneurysm; both an fSSV and a tSSV in 1 patient with an ICA-PCoA aneurysm; and both a tSSV and a TPV in 1 patient with a BX aneurysm. In all of these patients, the dural entrance of these veins was located just below the ACP or just below the sphenoid ridge on the anterior wall of the middle cranial fossa (Figs. 1-4A).

With the use of the SPST technique, the temporal dura propria in combination with the SPS was peeled off from the periosteal dura and inner cavernous membrane around the SOF. After removal of the ACPs, the incision of the dural flap stretched to the distal dural ring of the ICA; therefore, the bridging portion combined with the dural entrance of the obstructive bridging veins was displaced posteriorly (Figs. 1-4). Consequently, adequate surgical spaces around the lateral retrocarotid space were obtained without preservation of these veins in all patients. No perioperative complications associated with the SPST technique were observed in any patients, including venous infarction; neural injuries, including visual disturbances, ophthalmoplegia, and ptosis; and postoperative CSF leakage.

\section{Quantitative Analysis of the Working Area Before and After Surgery With the SPST Technique}

The mean \pm SD surface area of the lateral retrocarotid space was significantly increased from $18.3 \pm 18.8 \mathrm{~cm}^{2}$ before to $64.2 \pm 21.1 \mathrm{~cm}^{2}$ after the SPST technique was performed $(p=0.002$, Table 2). Exposure of the mean surface area of the aneurysm was significantly increased from $2.8 \pm 2.5$ to $20.9 \pm 20.6 \mathrm{~cm}^{2}(\mathrm{p}=0.001)$. Exposure of the length of the lateral aspect of the ICA did not differ from before $(7.9 \pm 4.3 \mathrm{~mm})$ to after $(14.9 \pm 3.9 \mathrm{~mm})$ the SPST technique was performed $(\mathrm{p}=0.007)$, although there was a trend toward a longer length after the SPST technique was performed.

\section{Discussion}

ICA-PCoA and BX aneurysms are approached via a route between the brain and the skull base. The SPST technique is superfluous for treating regular ICA-PCoA and BX aneurysms. However, in some cases, bridging veins running from the brain to the dural sinus that drains into the SPS restrict the surgeons' ability to obtain an adequate surgical field. In our series, the SPST technique was performed in such cases and enabled safe brain retraction without venous injury and markedly increased the surgical space around the lateral retrocarotid area.

TABLE 2. Comparison of parameters between the pre- and post-SPST techniques

\begin{tabular}{ccccc}
\hline Parameter & Pre-SPST & Post-SPST & Exposure Increase After SPST, \% & p Value* \\
\hline Retrocarotid space area, $\mathrm{cm}^{2}$ & $18.3 \pm 18.8$ & $64.2 \pm 21.1$ & 445 & 0.002 \\
\hline Aneurysm exposed area, $\mathrm{cm}^{2}$ & $2.8 \pm 2.5$ & $20.9 \pm 20.6$ & 161 & 0.001 \\
\hline ICA lateral aspect length, $\mathrm{mm}$ & $7.9 \pm 4.3$ & $14.9 \pm 3.9$ & 123 & 0.007 \\
\hline
\end{tabular}

Mean values are presented \pm SD.

${ }^{*}$ Mann-Whitney U-test. 
Although the rarity of the clinical complications caused by venous sacrifice may allow surgeons to sacrifice the SSVs and their tributaries, ${ }^{4,5}$ sacrificing these veins can cause venous congestion, hemorrhage, and edema. Dean et al. ${ }^{7}$ investigated the incidence of postoperative angiographic changes in the SSV and cerebral edema on postoperative CT after clipping surgery in 100 consecutive patients with middle cerebral artery aneurysms. In 11 cases with severely affected veins on postoperative angiography, postoperative CT detected cerebral edema with a mean range of $10.8 \mathrm{~cm}^{3}$. In this report, Dean et al. clarified that the damage to the venous outflow was correlated with the development of brain edema and small hemorrhages in the brain parenchyma. At present, there is no established method to predict the consequence of venous sacrifice; thus, surgeons should endeavor to preserve the SSVs and their tributaries while reserving a wide enough space within the surgical field for safe manipulation.

In the 8 patients in whom the SPST technique was performed in our series, the bridging segments of the obstructive bridging veins were relatively short, and these bridging veins entered the dural sinuses at a high position on the inner surface of the sphenoid ridge. These anatomical variations contributed to the alleviation of tension injury from brain retraction at the dural entrance of the sphenoid wing. Suzuki et al. ${ }^{6}$ investigated whether anatomical variations in SSVs affect the difficulty of surgical clipping via the pterional-transsylvian approach. In 8 of their 50 consecutive patients, the sylvian veins entered the dural sinuses at a relatively high position just below either the sphenoid ridge or the anterior clinoid process on the preoperative 3D-CTA images, and widening of the surgical corridor was restricted by these veins. Preoperative assessment of the draining position of the bridging veins near the sphenoid wing would be useful for predicting whether bridging veins obstruct the widening of the surgical space around the lateral retrocarotid space.

The peeling off of the arachnoid membrane that wraps the SSVs allows the vein to stretch, and freeing the veins from the brain enables the vein to be lifted when the brain moves away from the dura at the skull base.,11 These maneuvers were used in our series before the SPST technique was performed. However, in the cases in which the tributaries from the obstructive bridging veins were situated near the side of the skull base, the bridging segments of these veins were not stretched effectively with these manipulations. The SPST technique displaced these veins posteriorly and enabled the attainment of adequate surgical space.

With the SPST technique, the intradural segment of the obstructive bridging veins was separated from the skull base by peeling of the dura propria while maintaining the intradural segments of these veins on the side of the dura propria (Fig. 3C); therefore, the obstructive bridging veins were displaced posteriorly. A few studies have described venous preserving techniques in cases in which brain retraction can cause avulsion or tension injury at the dural entrance of the bridging vein. Kyoshima et al..$^{10}$ reported a method for obtaining mobility of the sylvian vein with intradural dissection of the dura propria of the cranial wall near the dural entrance of the SSV. Katsuno et al. ${ }^{3}$ also de- scribed a technique used in a modified anterior temporal approach for a BX aneurysm that elongated the bridging part of the sylvian vein by intradural dissection of the dura propria around the dural entrance of the sylvian vein. This maneuver is useful for stretching the bridging segment of the sylvian vein. However, the course of the intradural segment of the sylvian vein is less visible, and this method is often accompanied by intense bleeding due to injury of the intradural segment of the bridging vein, which may cause severe damage to the venous drainage. Hasegawa et al. ${ }^{12}$ reported a method for preserving an FBBV via mobilization of the SPS without removal of the ACP during clipping surgery for an anterior communicating aneurysm. Our method, in contrast, includes anterior clinoidectomies for extending a dural incision near the ICA, which facilitates further posterior displacement of the dural entrance of the bridging veins and widens the surgical space around the retrocarotid space.

The maneuver of peeling the dura propria within the correct layer is of utmost importance for accomplishing the SPST technique. If the temporal dura popria is peeled too shallowly, the sphenoparietal sinus coursing in the temporal dura propria can be damaged during peeling of the dura propria (Fig. 3C), which may lead to intense bleeding and/or severe venous congestion. In contrast, if the dura is peeled too deeply, cranial nerves (abducens and oculomotor, trochlear, and ophthalmic divisions of the trigeminal nerve) running together in the "inner" cavernous membrane ${ }^{13}$ around the SOF can be damaged. To avoid injury to these structures during peeling of the dura propria, it is vital to identify the correct layer between the dura propria and the periosteal dura and the inner cavernous membrane. In our series, a cleavage plane that appeared between the frontal and the temporal dura propria at the MOB (Fig. 1D) was helpful for estimating the thickness of the dura propria and the correct layer between the dura propria and the periosteal dura. In all of our patients, the SPST technique was conducted without injury to the SPS.

In our series, there were no cases of additional skull base modifications for BX aneurysms, such as those performed during orbitozygomatic osteotomy. Orbitozygomatic osteotomy allows excellent exposure of lesions involving the middle fossa, infratemporal region, and BX by decreasing the depth of the target and increasing the angle of approach. ${ }^{2,14,15}$ In clipping surgery for BX aneurysms, especially for high-riding BX aneurysms, orbitozygomatic osteotomy combined with the SPST technique would allow marked increases in the surgical space and greater maneuverability of instruments while lessening venous injury due to brain retraction.

\section{Study Limitations}

The current study is limited because it is a retrospective review of a small case series from a single institution. The SPST technique must not be interpreted as a routine approach to regular PCoA and BX aneurysms, because this procedure takes a longer time and requires more surgical expertise than does the regular transsylvian approach. In addition, this approach is technically slightly difficult. Intense bleeding as a result of injury of the SPS as well as cranial nerve injury, especially injury to the oculomotor 
nerve, may occur during peeling of the temporal dura propria. The optic nerve may also be injured when the anterior clinoid process is removed. ${ }^{16}$ The SPST technique should be performed in select situations by a surgeon with sufficient experience and expertise in this area.

\section{Conclusions}

The SPST technique, which can be used to mobilize the bridging veins between the brain and the dura on the inner surface of the sphenoid bone, facilitates widening of the surgical corridor. The current study is limited because it is a retrospective review of a small case series from a single institution. Notwithstanding, we believe that this maneuver is helpful for improving surgical outcomes by decreasing the risk of venous complications.

\section{References}

1. Sanai N, Caldwell N, Englot DJ, Lawton MT. Advanced technical skills are required for microsurgical clipping of posterior communicating artery aneurysms in the endovascular era. Neurosurgery. 2012;71(2):285-295.

2. Hsu FP, Clatterbuck RE, Spetzler RF. Orbitozygomatic approach to basilar apex aneurysms. Neurosurgery. 2005;56(1) (suppl):172-177.

3. Katsuno M, Tanikawa R, Izumi N, Hashimoto M. A modified anterior temporal approach for low-position aneurysms of the upper basilar complex. Surg Neurol Int. 2015;6:10.

4. Rhoton AL Jr. The cerebral veins. Neurosurgery. 2002;51(4) (suppl):S159-S205.

5. Lawton MT. Middle cerebral artery aneurysms. In: Seven Aneurysms: Tents and Techniques. New York, NY: Thieme Medical Publishers; 2011:65-93.

6. Suzuki Y, Nakajima M, Ikeda H, et al. Preoperative evaluation of the venous system for potential interference in the clipping of cerebral aneurysm. Surg Neurol. 2004;61(4):357364.

7. Dean BL, Wallace RC, Zabramski JM, et al. Incidence of superficial sylvian vein compromise and postoperative effects on CT imaging after surgical clipping of middle cerebral artery aneurysms. AJNR Am J Neuroradiol. 2005;26(8):20192026.

8. Kazumata K, Kamiyama H, Ishikawa T, et al. Operative anatomy and classification of the sylvian veins for the distal transsylvian approach. Neurol Med Chir (Tokyo). 2003;43(9):427-434.

9. Maekawa H, Hadeishi H. Venous-preserving sylvian dissection. World Neurosurg. 2015;84(6):2043-2052.
10. Kyoshima K, Oikawa S, Kobayashi S. Preservation of large bridging veins of the cranial base: technical note. Neurosurgery. 2001;48(2):447-449.

11. Sugita K, Kobayashi S, Yokoo A. Preservation of large bridging veins during brain retraction. Technical note. J Neurosurg. 1982;57(6):856-858.

12. Hasegawa $H$, Inoue $T$, Sato $K$, et al. Mobilization of the sphenoparietal sinus: a simple technique to preserve prominent frontobasal bridging veins during surgical clipping of anterior communicating artery aneurysms: technical case report. Neurosurgery. 2013;73(1)(Suppl Operative):E124-E127, ons128-ons129.

13. Umansky F, Nathan $H$. The lateral wall of the cavernous sinus. With special reference to the nerves related to it. $J \mathrm{Neu}$ rosurg. 1982;56(2):228-234.

14. van Furth WR, Agur AM, Woolridge N, Cusimano MD. The orbitozygomatic approach. Neurosurgery. 2006;58(1) (suppl):ONS103-ONS107.

15. Gonzalez LF, Crawford NR, Horgan MA, et al. Working area and angle of attack in three cranial base approaches: pterional, orbitozygomatic, and maxillary extension of the orbitozygomatic approach. Neurosurgery. 2002;50(3):550-557.

16. Matano F, Tanikawa R, Kamiyama H, et al. Surgical treatment of 127 paraclinoid aneurysms with multifarious strategy: Factors related with outcome. World Neurosurg. 2016;85:169-176.

\section{Disclosures}

The authors report no conflict of interest concerning the materials or methods used in this study or the findings specified in this paper.

\section{Author Contributions}

Conception and design: Niibo. Acquisition of data: Niibo, Takizawa, Takebayashi, Koizumi, T Kobayashi, R Kobayashi, Kuris, Gotou, Tsuchiya. Analysis and interpretation of data: Niibo, Sakurai. Drafting the article: Niibo. Study supervision: Kamiyama.

\section{Supplemental Information \\ Videos}

Video 1. https://vimeo.com/387953171.

\section{Correspondence}

Takeya Niibo: Asahikawa Red Cross Hospital, Asahikawa, Hokkaido, Japan.takeya.niibo@gmail.com. 